\title{
Molecular dynamics simulation of the effect of solute atoms on the compression of magnesium alloy
}

\author{
Qianhua Yang ${ }^{1} \cdot$ Chun Xue ${ }^{1} \cdot$ Zhibing Chu $^{1}$ (I) $\cdot$ Yugui Li ${ }^{1} \cdot$ Lifeng $\mathrm{Ma}^{2}$
}

Received: 20 April 2021 / Accepted: 25 May 2021 / Published online: 1 June 2021

(c) The Author(s) 2021

\begin{abstract}
Magnesium alloys have a wide range of application values. To design and develop magnesium alloys with excellent mechanical properties, it is necessary to study the deformation process. In this paper, the uniaxial compression (UC) process of AZ31 magnesium alloy with different solute atom content is simulated by the molecular dynamics method. The effect of the solute atom on the uniaxial compression of magnesium alloy is investigated. It is found that solute atoms can inhibit the grain refinement of magnesium, can effectively improve the plastic strength of the alloy, can change the lattice distortion during uniaxial compression of magnesium alloy, can inhibit the generation of BCC structure, and can slow down the increase of FCC structure and dislocation density. The direction of the FCC structure diffusion is $90^{\circ}$ to the grain boundary direction. Shockley partial dislocations are generated around the FCC structure. The direction in which the FCC structure spreads is consistent with the direction in which Shockley partial dislocations move.
\end{abstract}

Keywords Molecular dynamics $\cdot$ Magnesium alloy $\cdot$ Solute atom $\cdot$ Shockley partial dislocation

\section{Introduction}

As a green structural material in the twenty-first century, magnesium alloy has the advantages of low density, high specific strength, impact resistance, recyclability, and good heat dissipation performance. It has wide application value in many industries such as automobile, aerospace, medical, and transportation. As the alloy with the earliest development, the most grades, and the most widely used alloys, AZ series magnesium alloys have medium strength and high plasticity. Among them, AZ31 alloy has good strength and ductility, but it still produces internal structural defects during processing and deformation, which affects the mechanical properties. Therefore, studying the deformation process of magnesium alloy to improve its properties has become

Zhibing Chu

s20190347@stu.tyust.edu.cn

Qianhua Yang

1611758235@qq.com

1 College of Materials Science and Engineering, Taiyuan University of Science and Technology, Taiyuan 030024, China

2 College of Mechanical Engineering, Taiyuan University of Science and Technology, Taiyuan 030024, China an important research direction of magnesium alloy processing [1-7]. Because magnesium is a hexagonal closely packed (HCP) structure and has less slip system, its deformation mechanism is different from that of face-centered cubic (FCC) metal $[8,9]$. Nanocrystalline metals and alloys show different characteristic mechanical properties from coarse-grained metals and alloys. Compared with coarsegrained polycrystalline materials, nanocrystalline materials show higher strength, hardness, and toughness. Therefore, the nanostructure is an effective way to improve the strength and plasticity of magnesium alloys [10-15].

Most scholars study the influence of solute atoms on the metal matrix at the macro level. For example, Yu Daliang [16] used an optical microscope, scanning electron microscope, and universal testing machine to study the effect of composite addition of trace alloying elements on the microstructure and mechanical properties of AZ31 magnesium alloy. The results showed that the composite addition of trace alloying elements can improve the microstructure of AZ31 magnesium alloy, thus greatly improving the strength of magnesium alloy thus greatly increasing the strength. Huang Xiaofeng [17] studied the effect of $\mathrm{Zn}$ content on the as-cast and semi-solid microstructure of magnesium alloy through an optical microscope, scanning electron microscope, and universal mechanical testing machine. It was found that 
with the increase of $\mathrm{Zn}$ content, the as-cast microstructure of the alloy was refined, and the grain size of the semi-solid structure of the alloy was fine and round. Ma Yongdong [18] studied the effect of zinc content on the microstructure, mechanical properties, and damping properties of cast magnesium-zinc-zirconium alloy by microstructure observation, mechanical, and damping test. The results show that the compressive strength of $\mathrm{Mg}-\mathrm{Zn}-\mathrm{Zr}$ alloy increases with the increase of zinc content. Hou Shuaichang [19] adjusted the content of $\mathrm{Al}$ and $\mathrm{Zn}$ in the $\mathrm{AZ}$ magnesium alloy and found that as the content of $\mathrm{Al}$ and $\mathrm{Zn}$ increased, the strength of the extruded magnesium alloy increased, but the plasticity decreased.

In recent decades, with the tremendous increase in computing power, molecular dynamics simulation as a new computational material method has been successfully used to study lattice distortion, grain growth, deformation, and other aspects [20-24]. Some scholars have used molecular dynamics methods to study the influence of solute atoms [25-29] and found that solute atoms can affect the yield strength and dislocation formation of alloys. Molecular dynamics technology can effectively reproduce the mechanical behavior of materials at the atomic level, but few people use it to study the strength and deformation mechanism of nano-magnesium alloys [30].

In this paper, to explore the effect of solute atom content on AZ31 magnesium alloy, the model of AZ31 magnesium alloy with different solute atom content was established by Atomsk, the uniaxial compression (Uniaxial Compression, UC) process of AZ31 magnesium alloy was simulated by molecular dynamics method, and the mechanical properties and structural changes of AZ31 magnesium alloy during UC were analyzed by OVITO, which provides a theoretical basis for the design and development of nanocrystalline AZ31 magnesium alloy with excellent mechanical properties.

\section{The simulation method}

First of all, the nanocrystalline Mg model with the size of $192.6 \AA \times 222.395 \AA \times 208.4 \AA$ is established by Atomsk. The number of atoms is 384020 , and the initial number of grains is 20 . The $\mathrm{X}$-axis, $\mathrm{Y}$-axis, and $\mathrm{Z}$-axis correspond to the crystal directions of $[\overline{1} 2 \overline{1} 0],[\overline{1} 010]$ and [0001], respectively. Then, the corresponding magnesium atoms are replaced according to Fig. 1 [31]. The polycrystalline samples of pure magnesium are named $\mathrm{NC} \mathrm{Mg}$, and the AZ31 magnesium alloy specimens with different solute atomic contents are named Alloy $1 \sim 5$.

The potential function is a mathematical function form describing the total energy $\mathrm{E} E$ of the model particles and the particle coordinates $(r)(r)$ [32-35]. The embedded atom

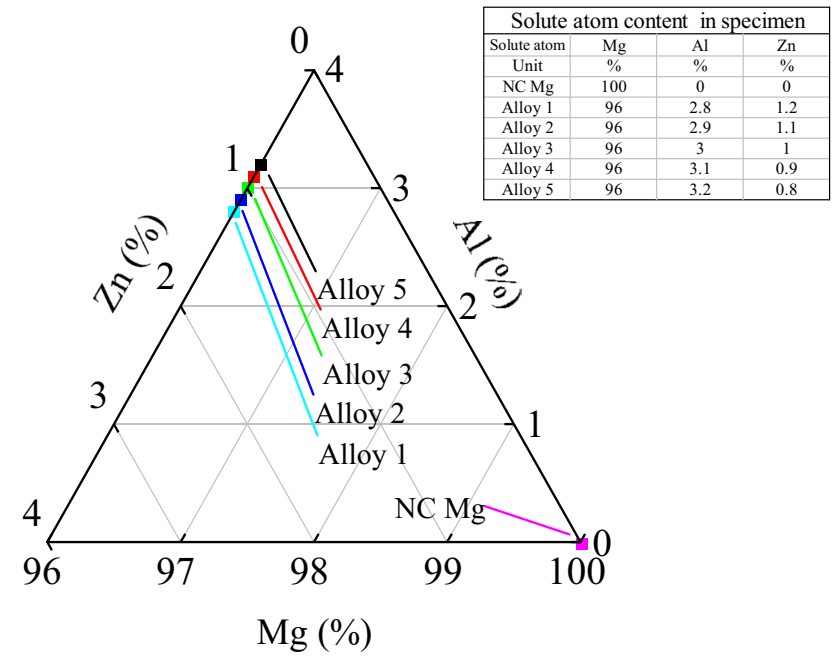

Fig. 1 The content of each element in the specimens

method (EAM) potential is usually used to calculate the pairwise interaction between metal and metal alloy. The improved embedded atomic method (MEAM) potential can not only calculate the pairwise interactions of various materials but also be suitable for modeling metals and alloys with FCC, BCC, HCP, and Diamond structures and covalently bonded materials such as silicon and carbon [33-42]. In the MEAM formula, the total energy $\mathrm{E} E$ of the atomic system is

$E=\sum_{i}\left\{F_{i}\left(\bar{\rho}_{i}\right)+\frac{1}{2} \sum_{i \neq j} \phi_{i j}\left(\gamma_{i j}\right)\right\}$

In the formula, $\mathrm{E} E$ is the total energy of atomic $i . F$ is the embedded energy, which is a function of atomic electron density $\rho . \rho$ is atomic electron density. $\gamma$ and $\phi$ are a pair of potential interactions. And $i$ and $j$ are element types.

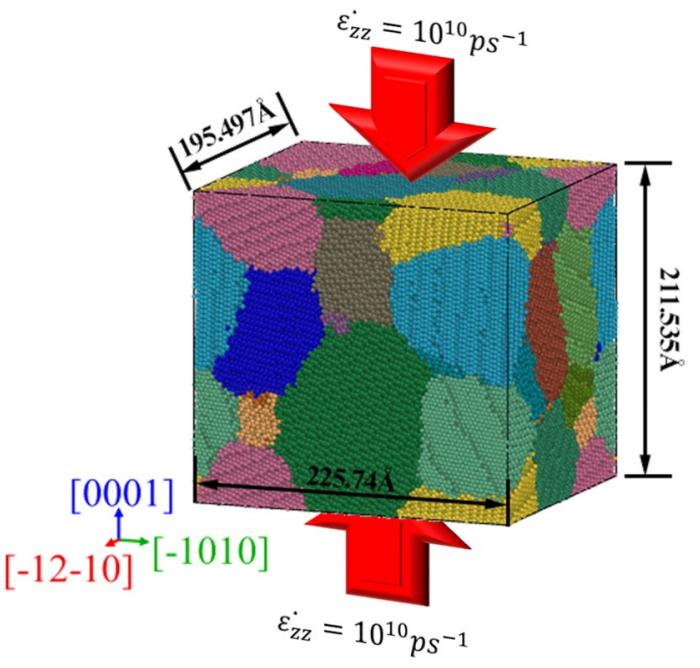

Fig. 2 Schematic diagram of compression deformation 
The established sample model is imported into LAMMPS (large-scale atomic/molecular massively parallel simulator) [43, 44], and the $\mathrm{Mg}-\mathrm{Al}-\mathrm{Zn}$ potential function developed by Hyo-Sun Jang [45] is assigned to the model. This potential function can be used to study the deformation and recrystallization properties of $\mathrm{Mg}-\mathrm{Al}-\mathrm{Zn}$ alloy in the whole processing temperature range. $X, Y$, and $Z$ are all periodic boundary conditions, the time step is $0.001 \mathrm{ps}$ and the ambient temperature is $300 \mathrm{~K}$. First, the NPT ensemble is used to relax the 10 ps under constant temperature and pressure. After relaxation, the system uses the NPT ensemble and deform compression combination command to uniformly compress $20 \mathrm{ps}$ at the strain rate of $0.01 \mathrm{ps}^{-1}$ along the $Z$-axis ([0001] crystal direction), and the final deformation of the magnesium alloy reaches $20 \%$, that is, through the comparative feedback between the stress of the calculation system and the set stress, the unit cell length is automatically adjusted, and then the strain is calculated. The process diagram is shown in Fig. 2.

\section{Simulation results and discussion}

The simulation results are imported into the visualization software OVITO, and the functions of common neighbor analysis (CNA), dislocation analysis (DXA), polyhedral template matching (PTM), grain segmentation (GS), and Wigner-Seitz defect analysis (WSDA) are used for
Fig. 3 Grain changes of magnesium alloy specimens

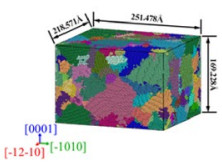

(a)Specimen 1

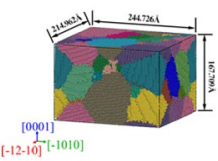

(d)Specimen 4

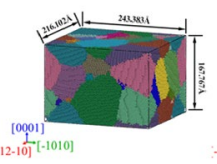

(b)Specimen 2

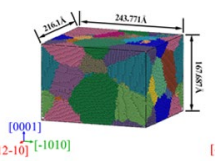

(e)Specimen 5

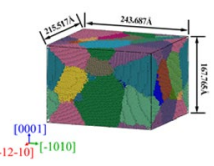

(c)Specimen 3

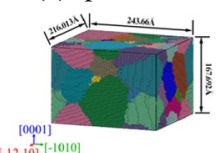

(f)Specimen 6

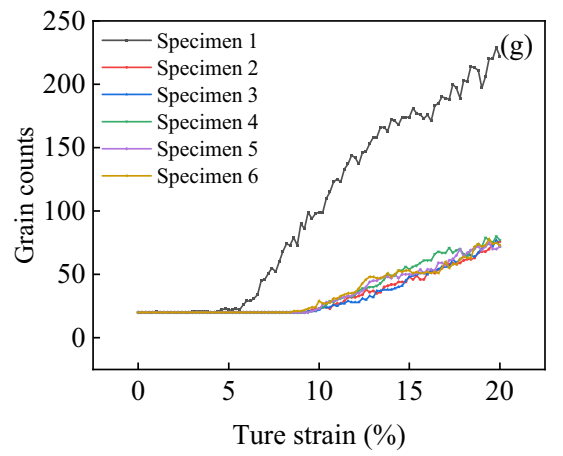

Fig. 4 Change of properties of magnesium alloy during compression. a Stress-strain curve; b volume change curve; c interstitial atom number curve; d dislocation density curve
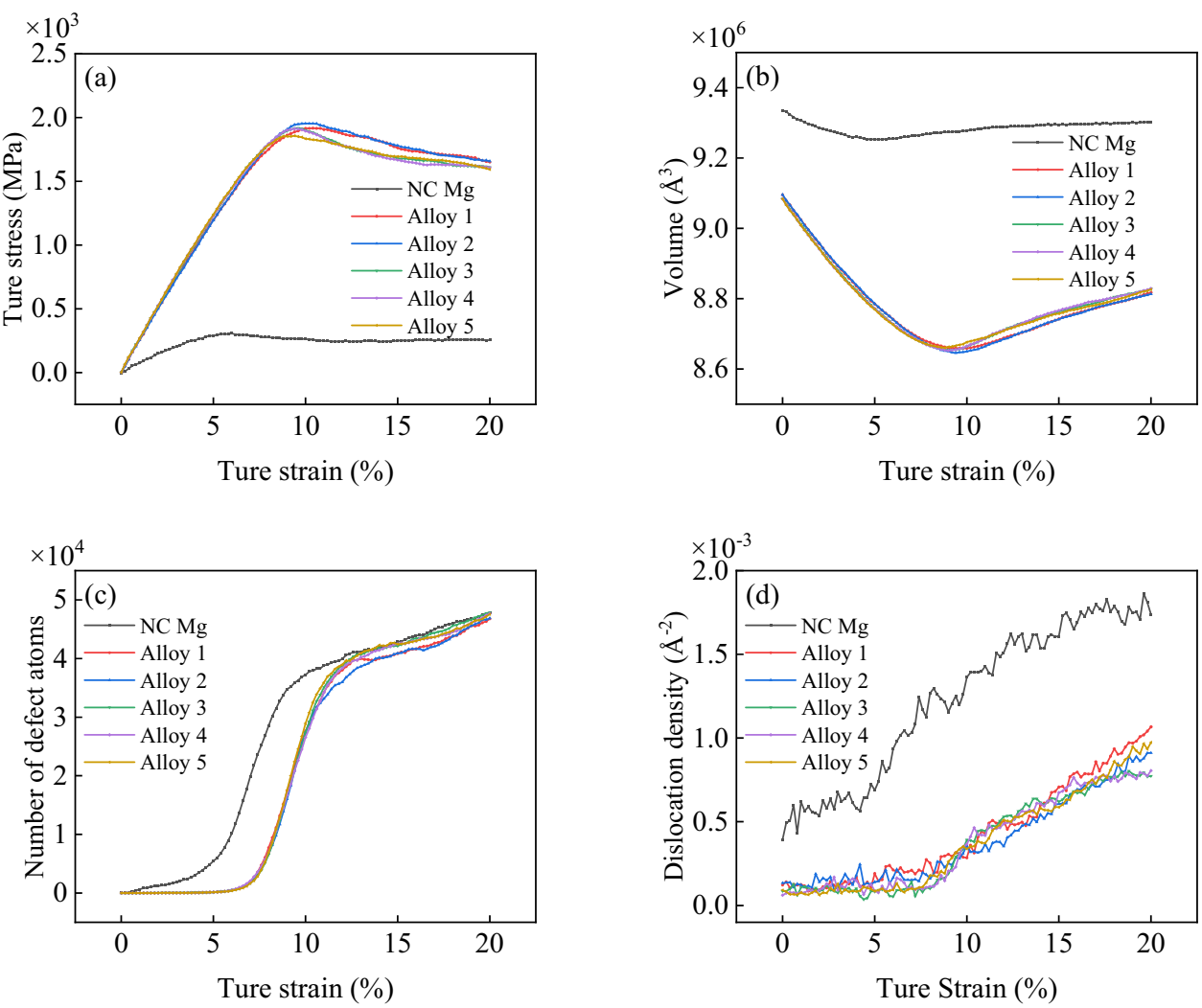

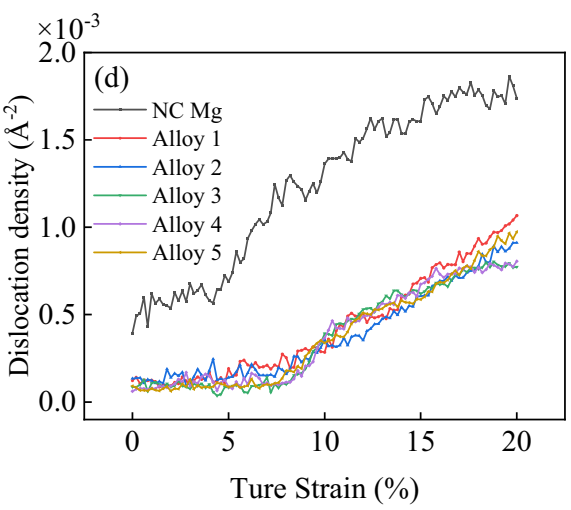


(a)

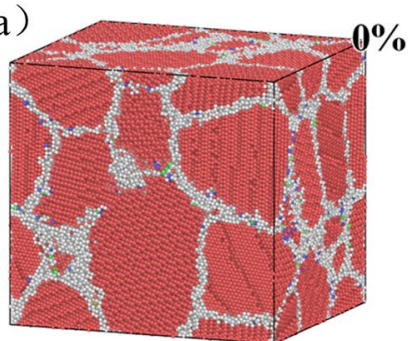

(b)

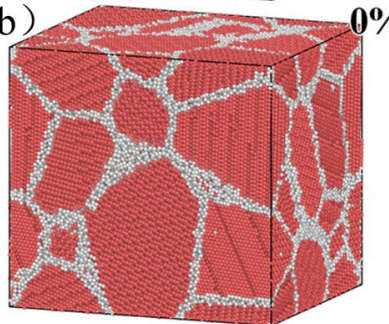

(c)

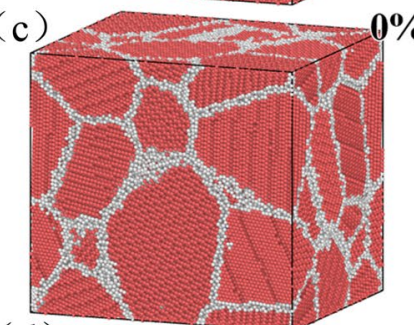

(d)

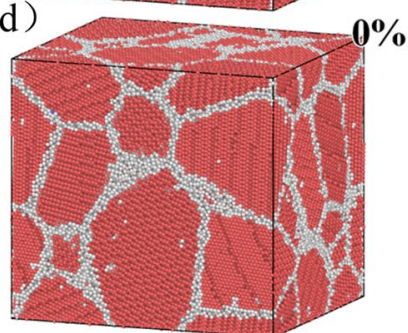

(e) $0 \%$

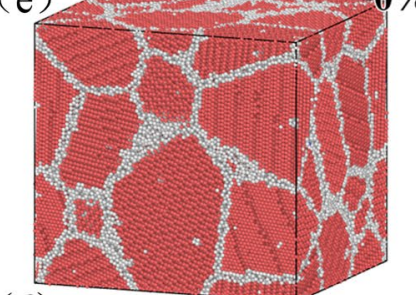

(f)

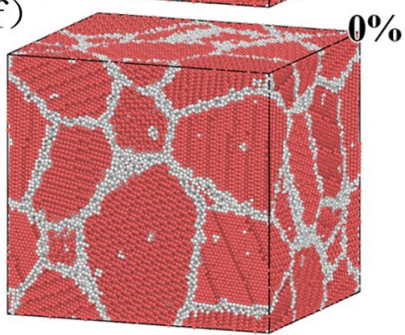

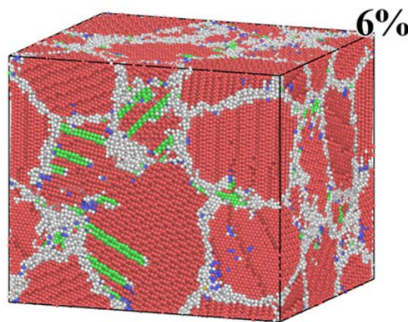
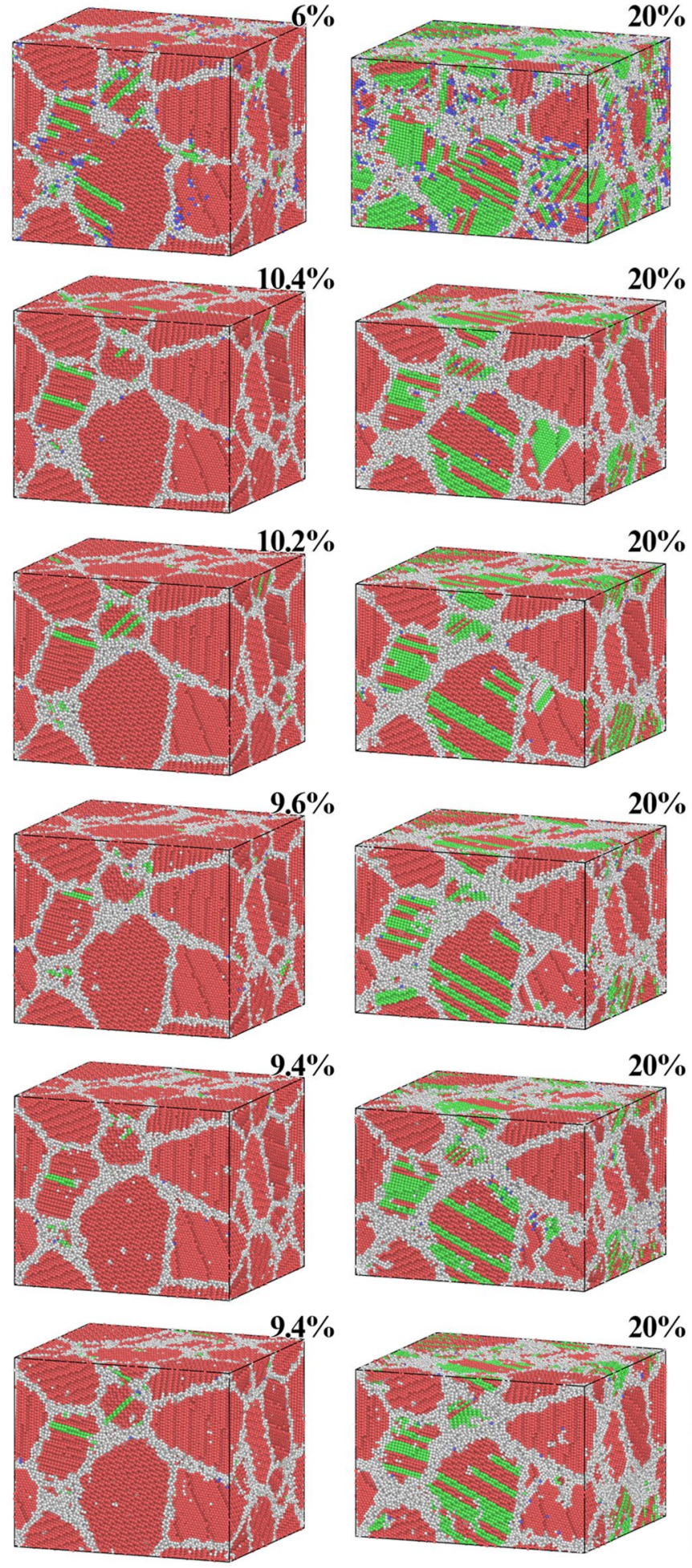

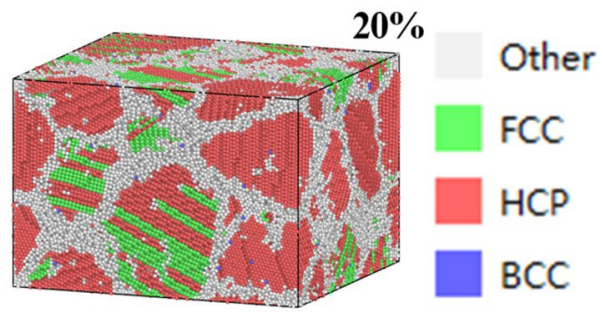

Fig. 5 CNA snapshot of alloy

analysis. CNA provides visualization of common crystal structures in metals, such as face-centered cubic (FCC), body-centered cubic (BCC), hexagonal closely packed (HCP) structure, and other structure (grain boundary atoms). The grain changes in the simulation process can be observed by GS. WSDA can identify the crystal void and calculate the number of interstitial atoms [46-51]. 
Figure $3 \mathrm{a}-\mathrm{f}$ is the grain distribution in the magnesium alloy specimens when $\varepsilon=20 \%$. Figure $3 \mathrm{~g}$ shows the grain number curve. It can be seen that the grain refinement of AZ31 decreases after the addition of solute atoms, and the number of grains decreases significantly after UC, so solute atoms can inhibit the grain refinement of $\mathrm{Mg}$.

Figure 4a shows the stress-strain curve of magnesium alloy during compression. It can be seen that in the stage of elastic deformation, the stress-strain curve maintains a linear relationship until it reaches the tensile strength when the stress reaches the maximum. With the increase of the amount of compression, the stress decreases sharply when it enters the stage of plastic deformation. The addition of solute atoms significantly increased the yield strength and elastic modulus of the alloy, and the ultimate strain increased by $173.33 \%, 170 \%, 160 \%, 156.67 \%$, and $156.67 \%$, respectively, indicating that solute atoms can effectively improve the plastic strength of the alloy.

Figure $4 \mathrm{~b}$ is the volume change curve, Fig. $4 \mathrm{c}$ is the interstitial atom quantity curve, and Fig. $4 \mathrm{~d}$ is the dislocation density curve. It can be seen that with the increase of compression amount, the atomic position in the alloy changes, the number of interstitial atoms increases gradually, the sample volume decreases gradually, and the dislocation density increases. When the amount of compression reaches UTS\% (the ultimate strain at the maximum yield strength), the increasing trend of the number of interstitial atoms slows down, and the lattice distortion occurs in the sample. With the increase of the amount of compression, the lattice distortion becomes more severe and the volume increases
Fig. 6 Potential energy and structure fraction curve
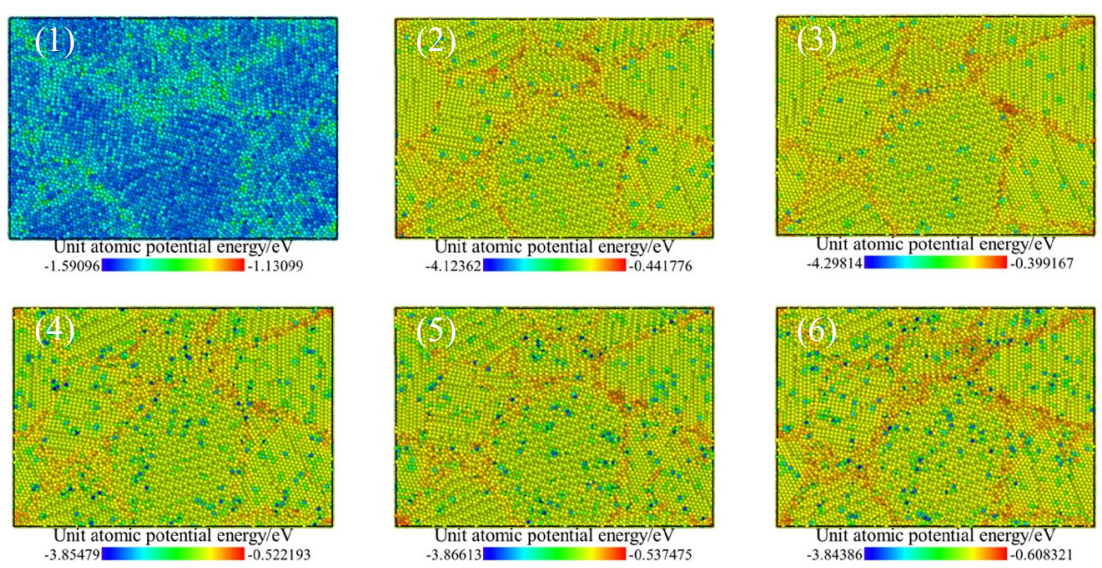
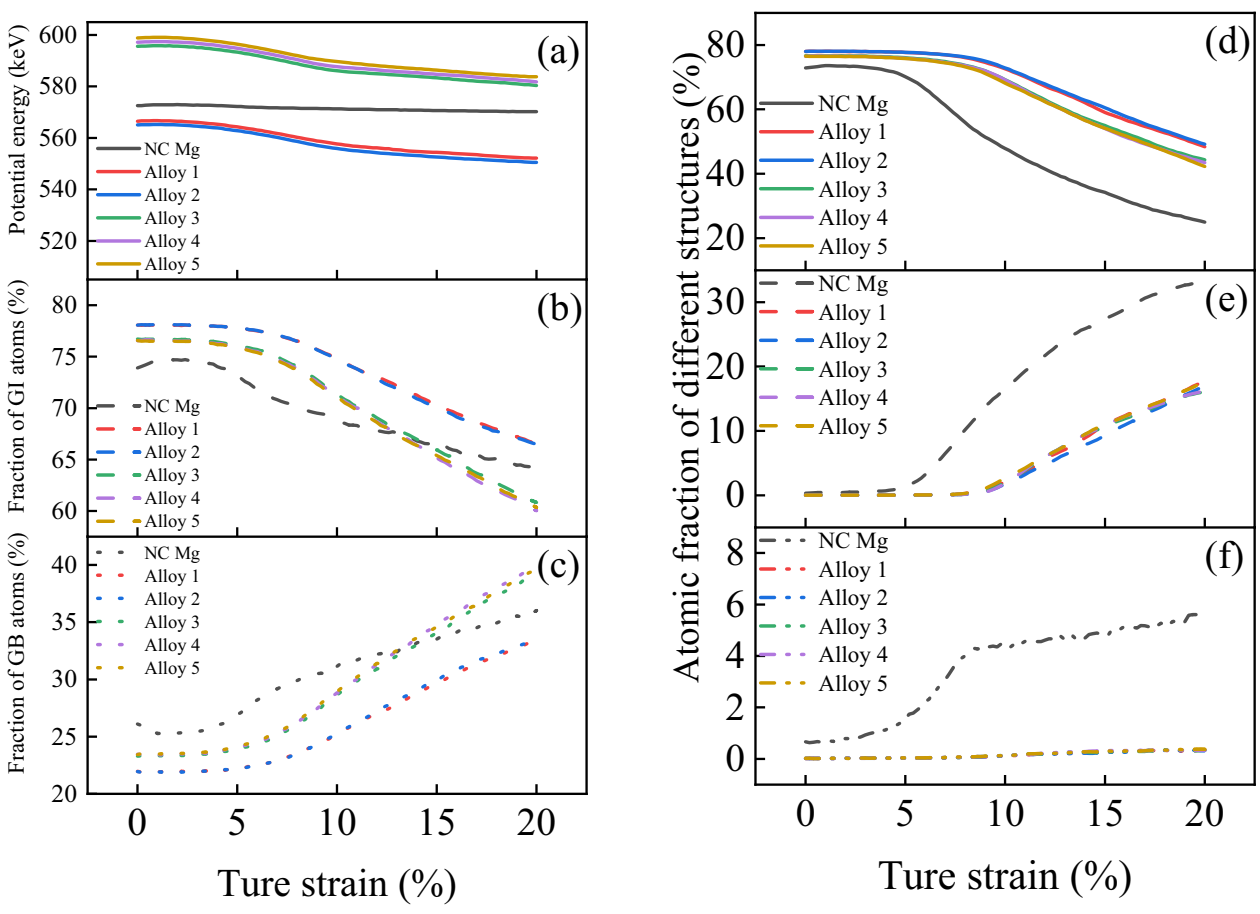

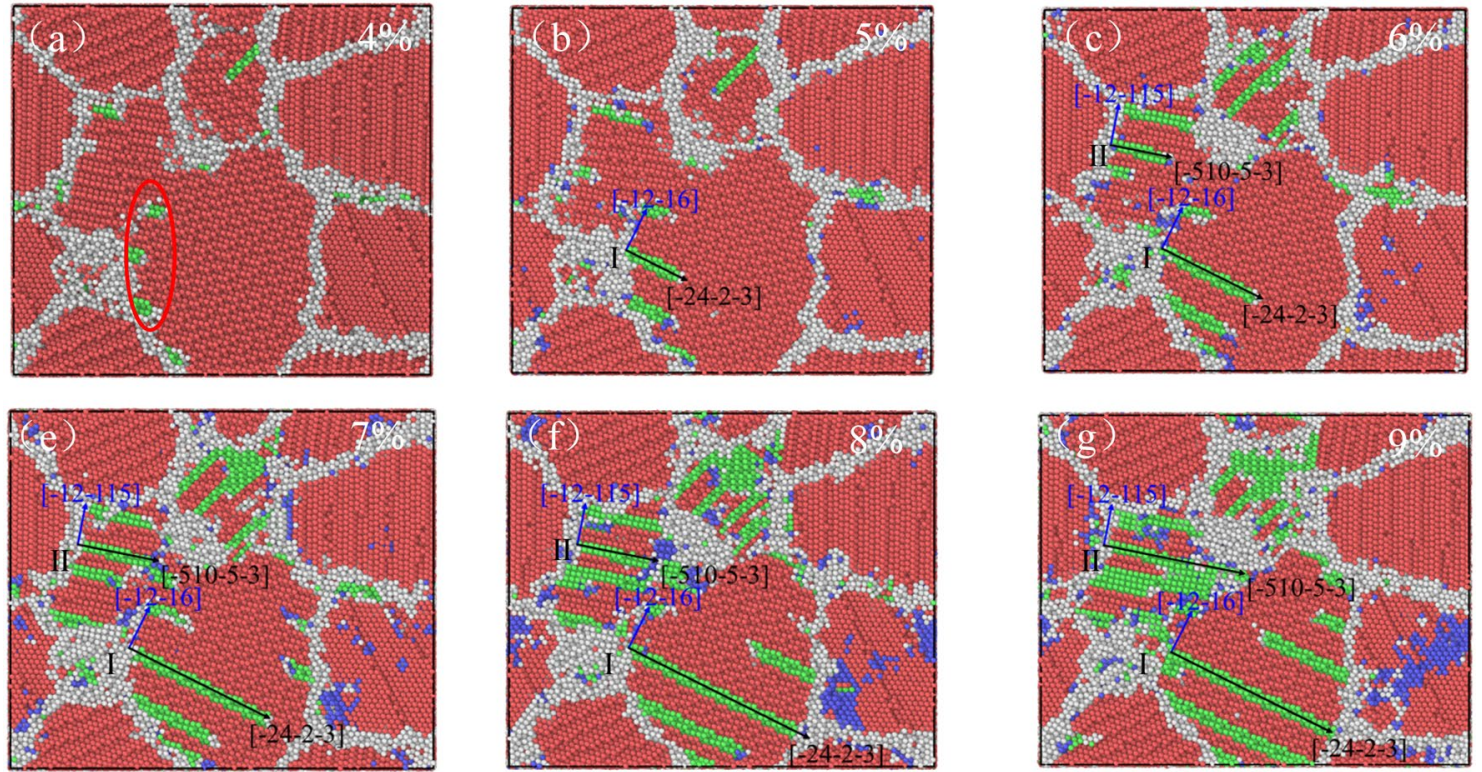

Fig. 7 FCC structure growth law

(a)

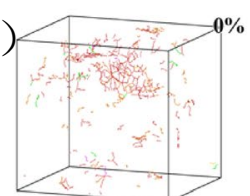

(b)

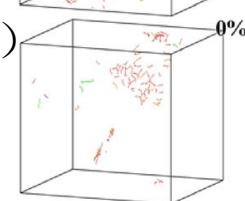

(c)

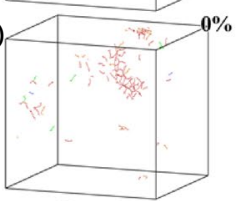

(d)

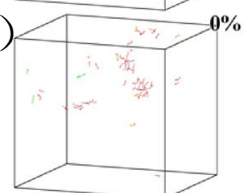

(e)

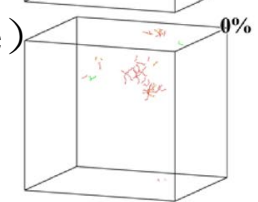

(f)

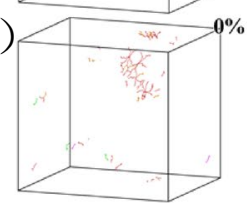

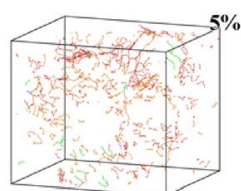
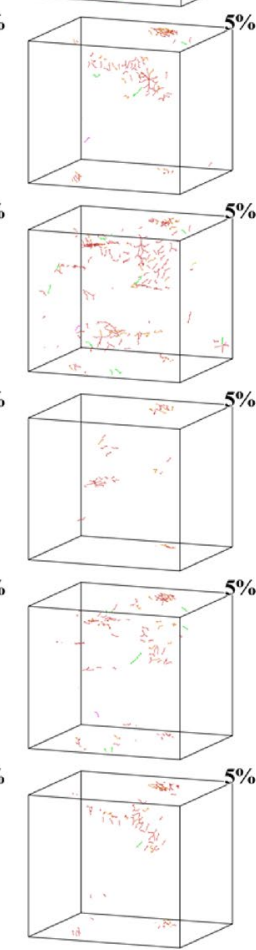
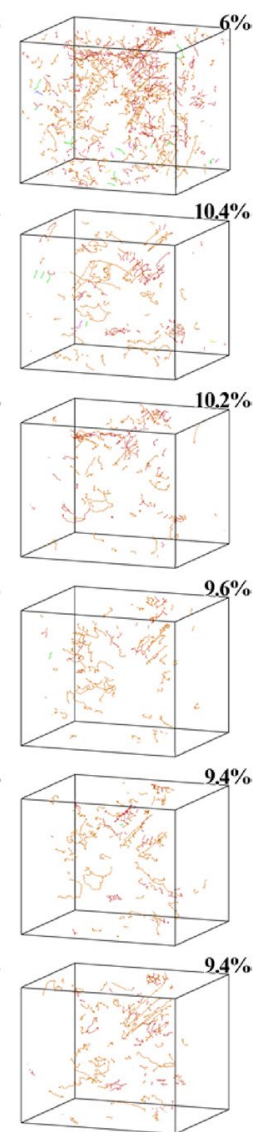
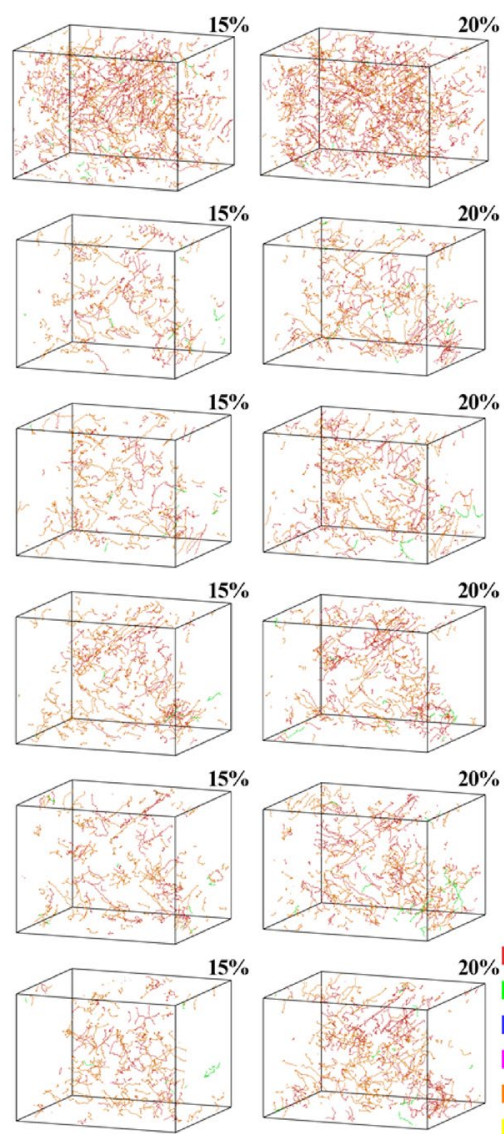

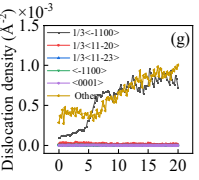

$\times 10^{-3}$ Ture Strain (\%)

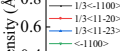

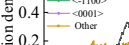

总 0.2 - Other

$\times 10^{-3}$ Ture Strain (\%)

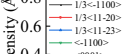

0.4 - conoly

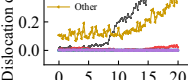

$T^{\times 10^{-3}}$ Ture Strain (\%)

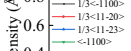

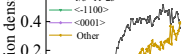

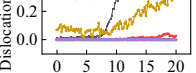

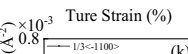

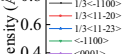

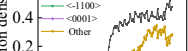

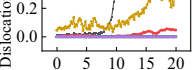

$\times 10^{-3}$ Ture Strain (\%)

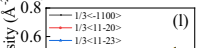

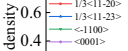

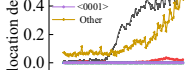

$<1-100>$

$1 / 3<1-100>$

Fig. 8 Dislocation growth change 
gradually. Compared with $\mathrm{NC} \mathrm{Mg,} \mathrm{the} \mathrm{addition} \mathrm{of} \mathrm{solute}$ atoms delays the increase of interstitial atoms, increases the trend of volume change, slows down the phase transition, and reduces the dislocation density, so the yield strength and elastic modulus of magnesium alloy are higher than those of $\mathrm{NC} \mathrm{Mg}$.

Figure 5 shows CNA snapshots of alloys with different solute atomic contents at $\varepsilon=0 \%$, UTS $\%, 20 \%$, respectively (The atoms in the model have been colored according to CNA results, red is HCP structure, green is FCC structure, blue is BCC structure, white is Other structure), Fig. 5a is $\mathrm{NC} \mathrm{Mg}$, Fig. $5 \mathrm{~b} \sim \mathrm{f}$ is Alloy $1 \sim 5$, respectively. It can be seen that with the progress of compression, the stress increases gradually with the strain and reaches its tensile strength, the stress concentration leads to more intense thermal movement of atoms, a large number of atoms are out of equilibrium, resulting in lattice distortion, HCP structure is gradually destroyed, some atoms from disorder to order, some atoms return to the equilibrium position, forming a new ordered lattice structure, becoming FCC structure and BCC structure. In the process of $\mathrm{NC} \mathrm{Mg}$ compression, a large number of FCC structure and a small amount of BCC structure are formed. After adding solute atoms, the BCC structure is almost not produced, the number of FCC structure formation is also significantly reduced, and the transformation is delayed, so solute atoms can change the lattice distortion of magnesium alloy during uniaxial compression.

Figure 6 (1)-(6) is a snapshot of the unit atomic potential energy. It can be seen that the atomic potential energy at the grain boundary is higher than that of the atoms in the grain. Figure $6 \mathrm{a}$ is the potential energy curve, Fig. $6 \mathrm{~b}$ is the intragranular atom (Grain Interior, GI) fraction curve, and Fig. $6 c$ is the grain boundary atom (Grain Boundary, GB) fraction curve. It can be seen that when $\varepsilon=20 \%$, when the atomic content of $\mathrm{Al}$ is more than $3 \%$, the atomic fraction of the grain boundary increases and the potential energy of the alloy exceeds NC Mg because of the high atomic potential energy at the grain boundary. With the increase of the amount of compression, the position of the atom changes, the potential energy decreases, the lattice distortion occurs, the atomic fraction of the grain boundary increases and the grain boundary diffuses. Figure $6 \mathrm{~d}-\mathrm{f}$ shows the atomic fraction curves of HCP, FCC, and BCC structures of the alloy during compression. Some of the atoms in $\mathrm{NC} \mathrm{Mg}$ have a $\mathrm{BCC}$ structure, and the BCC structure increases with the increase of compression. When $\varepsilon=\mathrm{UTS} \%$, the lattice distortion of $\mathrm{HCP} \rightarrow \mathrm{FCC}$ begins to occur, while after the addition of solute atoms, the BCC structure is almost not produced, so the solute atoms have a significant effect on the inhibition of BCC structure.

Since the grain boundary is relatively high, the layered FCC structure is first formed at the grain boundary (shown in the red elliptical ring shown in Fig. 7a), then grows inside the grain (as shown in Fig. 7b. The black arrow is shown, gradually throughout the grain. At I, the growth direction of the FCC phase is $[\overline{2} 4 \overline{23}]$, the grain boundary direction is $[\overline{1} 2 \overline{1} 6]$. At II, the growth direction of the FCC phase is
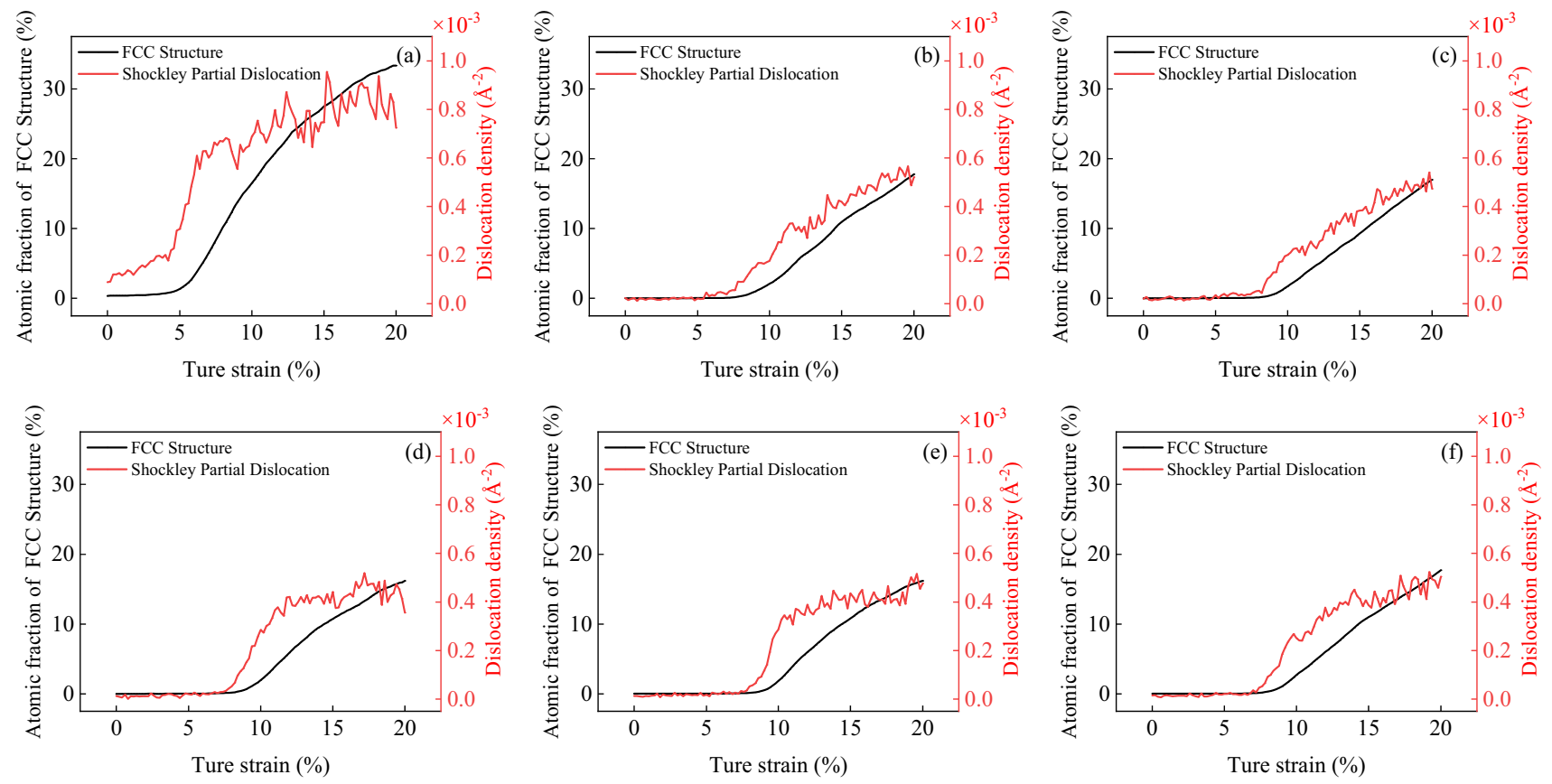

Fig. 9 the curve of FCC structure and Shockley partial dislocation 
$[\overline{5} 10 \overline{53}]$, the grain boundary direction is $[\overline{1} 2 \overline{1} 15]$. After calculation, it is found that the growth direction of the FCC structure is $90^{\circ}$ to the grain boundary direction.

Figure $8 \mathrm{a}$ shows the growth of dislocations in $\mathrm{NC} \mathrm{Mg}$, Fig. 8b-f shows the growth of dislocations in Alloy 1-5, and Fig. $8 \mathrm{~g}-1$ shows the dislocation growth curve. It can be seen that with the increase of compression, the dislocation density increases gradually, among which the most obvious increases are other dislocations and $1 / 3<--100>$ dislocations (Shockley partial dislocations) [52] the addition of solute atoms slows down the increase of FCC structure and dislocation density.

Figure 9 shows the curve of FCC structure and Shockley partial dislocations. It can be found that with the increase of reduction, the growth rate of FCC structure is almost the same as that of Shockley partial dislocations, so it is speculated that the changes of FCC structure and Shockley partial dislocations increase in positive proportion, and Shockley partial dislocations are attached to the FCC structure.

Figure 10 is a snapshot of the FCC structure and Shockley partial dislocations. As shown by the red circle in Fig. 10a, Shockley partial dislocations surround the FCC structure. The alloy is compressed and the position of the atoms changes, resulting in Shockley partial dislocations. Dislocations form dislocation plugging, and lattice distortion occurs near this position, and the HCP structure is transformed into the FCC structure. As the amount of compression increases, the positions of more atoms change, some of the Shockley dislocations move, and the FCC structure diffuses, and the direction of the FCC structure diffusion (yellow arrow in Fig.) and the direction of the Shockley part of the dislocation movement (Red arrow in the Fig.) consistent.

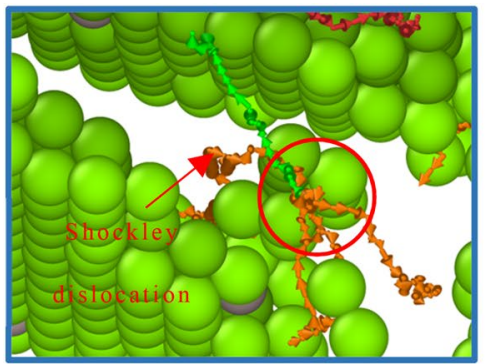

(a) $18.4 \%$

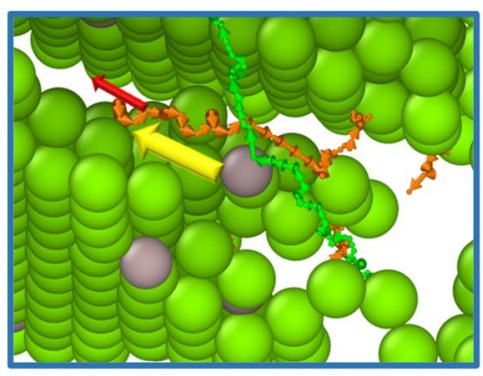

(d) $19 \%$

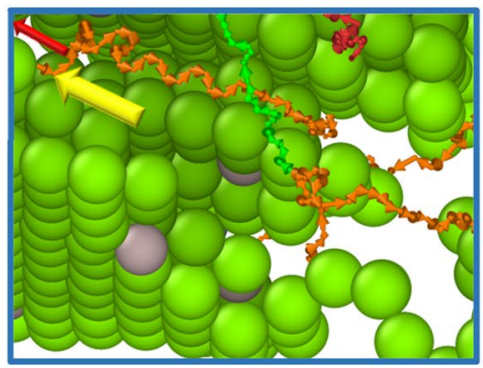

(g) $19.6 \%$

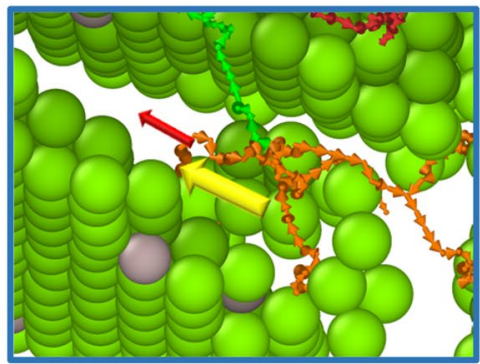

(b) $18.6 \%$

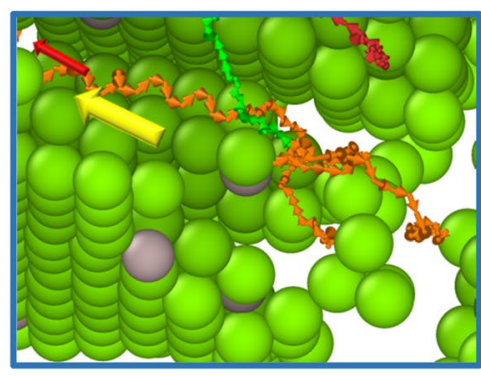

(e) $19.2 \%$

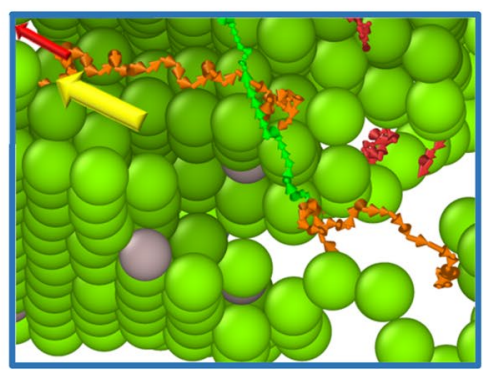

(h) $19.8 \%$

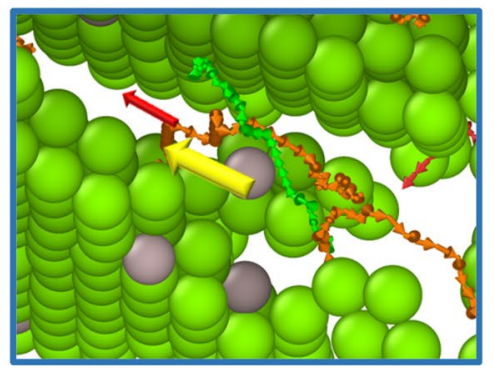

(c) $18.8 \%$

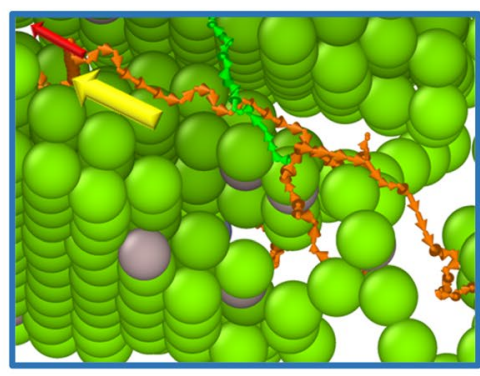

(f) $19.4 \%$

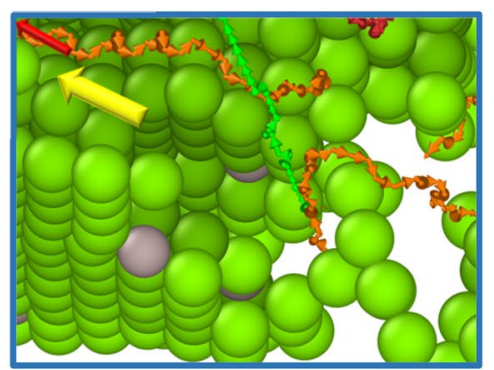

(i) $20 \%$

Fig. 10 Snapshot of FCC structure and Shockley partial dislocations 


\section{Conclusion}

This paper simulates the uniaxial compression of an AZ31 magnesium alloy of different solute atoms by molecular dynamics and explores the effects of solute atoms in magnesium alloy single shaft compression. The following conclusions are obtained:

(1) The solute atom can inhibit the grain refinement of magnesium, effectively improve the plastic strength of the alloy.

(2) The solute atom can change the lattice distortion during the uniaxial compression of the magnesium alloy, and there is a significant role in the suppression of the BCC structure, slowing the increase of the FCC structure and the dislocation density.

(3) The direction of the growth of the FCC structure is $90^{\circ}$ in the grain boundary direction, and the Shockley partial dislocations are hosted around the FCC structure, and the direction of the FCC structure diffuses is consistent with the Shockley partial dislocations.

Acknowledgements This work was supported by the National Key Research and Development Project (2018YFB1307902), National Science Fund Subsidized Project (No. U1710113), Shanxi Province Joint Student Training Base Talent Training Project (No. 2018JD33), Shanxi young top talent project, Shanxi Province Science Foundation for Youths (201901D211312), Excellent young academic leaders in Shanxi colleges and universities (No. 2019045), Excellent Achievements Cultivation Project of Shanxi Higher Education Institutions (No. 2019KJ028), Shanxi Province emerging industry leader talent project, Shanxi Graduate Education Innovation Project (No. 2019SY482).

\section{Declarations}

Conflict of interest The authors declare that they have no conflict of interest.

Open Access This article is licensed under a Creative Commons Attribution 4.0 International License, which permits use, sharing, adaptation, distribution and reproduction in any medium or format, as long as you give appropriate credit to the original author(s) and the source, provide a link to the Creative Commons licence, and indicate if changes were made. The images or other third party material in this article are included in the article's Creative Commons licence, unless indicated otherwise in a credit line to the material. If material is not included in the article's Creative Commons licence and your intended use is not permitted by statutory regulation or exceeds the permitted use, you will need to obtain permission directly from the copyright holder. To view a copy of this licence, visit http://creativecommons.org/licenses/by/4.0/.

\section{References}

1. X. Chen, Research progress in magnesium alloys as functional materials. Rare Metal Mater. Eng. 45(9), 2269-2274 (2016)
2. B. Ye, B. He, Research progress of high strength magnesium alloy and its preparation technology. Hot Working Technol 48(24), 5-10 (2019)

3. F. Abouhilou, A. Hanna, H. Azzeddine et al., Microstructure and texture evolution of AZ31 Mg alloy after uniaxial compression and annealing. J Magnesium Alloys 7(1), 124-133 (2019)

4. Zhongju $\mathrm{Hu}$, Yanfeng Liu, Liwei Lu, et al. Forward extrusion bending shear composite continuous deformation process and extrusion force calculation of magnesium alloy. Chin. J. Nonferrous Metals. 28(5):923-931 (2018).

5. J. Dang, Z. Jiang, L. Ren et al., A study on microstructure and hot deformation behavior of As-extruded AZ63M magnesium alloy. Rare Metal Mater Eng. 47(4), 1293-1301 (2018)

6. X. Chen, Q. Li, Y. Fan, Research status of AZ31 magnesium alloy. Shanxi Metallurgy 01, 1-3 (2009)

7. Wenxian Li. Magnesium and magnesium alloys. Central South University Press. (2005)

8. J. Wang, J.P. Hirth, C.N. Tomé, Twinning nucleation mechanisms in hexagonal-close-packed crystals. Acta Mater. 57(18), 5521-5530 (2009)

9. Kenneth Kanayo Alaneme, Eloho Anita Okotete. Enhancing plastic deformability of $\mathrm{Mg}$ and its alloys-A review of traditional and nascent developments. J Magnesium Alloys. 5(4):460-475 (2017).

10. H. Gleiter, Nanostructured materials: basic concepts and microstructure. Acta Mater. 48(1), 1-29 (2000)

11. Xu. Wenwu, X. Song, Lu. Nianduan et al., Thermodynamic and experimental study on phase stability in nanocrystalline alloys. Acta Mater. 58(2), 396-407 (2010)

12. X. Song, Y. Gao, X. Liu et al., Effect of interfacial characteristics on toughness of nanocrystalline cemented carbides. Acta Materialia. 61(6), 2154-2162 (2013)

13. C. Suryanarayana, Structure and properties of nanocrystalline materials. Bull. Mater. Sci. 17, 307-346 (1994)

14. Yanhao Dong, I. Wei Chen. Onset Criterion for Flash Sintering. J Am Ceramic Soc, 98, 3624-3627 (2018).

15. A. Mahata, K. Sikdar, Molecular dynamics simulation of nanometer-scale mechanical properties of hexagonal $\mathrm{Mg}$ single bond $\mathrm{Li}$ alloy. J Magnesium Alloys. 4(1), 36-43 (2016)

16. Daliang Yu, Qingwei Dai, Sensen Chai, et al. Effect of trace elements on Microstructure and mechanical properties of AZ31 magnesium alloy. J Mater Heat Treatment. 41(9),91-95 (2020)

17. Xiaofeng Huang, Cuicui Hao, Kai Feng, et al. The effect of $\mathrm{Zn}$ content on the microstructure of Mg-9Al magnesium alloy. New Technol New Process. (12):98-100 (2014).

18. Yongdong Ma, Anxia Pan, Liqun Ma, et al. Effect of $\mathrm{Zn}$ content on damping and mechanical properties of cast $\mathrm{Mg}-\mathrm{Zn}-\mathrm{Zr}$ alloy. Mech Eng Materials. 37(2):21-24,28 (2018).

19. Suaichang Hou. Effect of $\mathrm{Al}$ and $\mathrm{Zn}$ contents on Microstructure and mechanical properties of extruded and rolled mg Al Zn magnesium alloys. Jilin University (2016).

20. G.P. Zheng, Y.M. Wang, M. Li, Atomistic simulation studies on deformation mechanism of nanocrystalline cobalt. Acta Mater. 53(14), 3893-3901 (2005)

21. Xinghui Wu. A course of modern material calculation and design. Electronic Industry Press (2002).

22. Hui Wang, Yuanzhong Hu, Hun Zou, et al. Molecular dynamics simulation of nanotribology. Chinese Sci (Series A).31(3), 261-266 (2001).

23. Z. Jia, G. Yang, Z. Cheng et al., Molecular dynamics simulation of atomic behavior in $\mathrm{Si}(001)$ surface layer and near surface layer. Acta Physica Sinica. 43(4), 609-615 (1994)

24. Daiyu Zhang, Fusheng Liu, Xijun Li, et al. Molecular dynamics simulation of impact temperature of porous iron. J High Pressure Phys. (01):17-22 (2003). 
25. Sara Fazeli, Morteza Vahedpour, Sayed Khatiboleslam Sadrnezhaad. Effect of copper content on tensile mechanical properties of ternary NiTiCu alloy nanowire: Molecular dynamics simulation. Materials Today: Proceedings. 5(1):1552-1555 (2018).

26. Wu. Ronghai, Q. Yin, J. Wang et al., Effect of Re on mechanical properties of single crystal Ni-based superalloys: Insights from first-principle and molecular dynamics. J. Alloy. Compd. 862, 158643 (2021)

27. Sandeep Kumar Singh, Avinash Parashar. Defect dynamics and uniaxial tensile deformation of equi and non-equi-atomic configuration of multi-elemental alloys. Materials Chem Phys. 124549 (2021).

28. N. Razmara, R. Mohammadzadeh, Effect of nitrogen content on the crack growth behavior in the Fe-N alloy at high temperatures via molecular dynamics simulations. Theoret. Appl. Fract. Mech. 97, 30-37 (2018)

29. B. Zhang, X. Zhang, C. Li, Molecular dynamics simulation on phase transformation of Ti-Al Alloy with low Al content. Rare Metal Mater Eng.. 41(6), 1010-1015 (2012)

30. A. Moitra, Grain size effect on microstructural properties of 3D nanocrystalline magnesium under tensile deformation. Comput. Mater. Sci. 79, 247-251 (2013)

31. J. Zhou, Wu. Rendong, C. Yuan et al., Hot compression deformation behavior of AZ31B Magnesium Alloy under different processing conditions. Rare Metal Mater Eng. 406(05), 329-334 (2020)

32. Lu Xia, Song Chen, Jiansheng Lu, et al. Application and development of noble metal potential function for molecular dynamics simulation. Noble Metal. 4:82-90 (2018).

33. A. Caro, D.A. Crowson, M. Caro, Classical Many-Body Potential for Concentrated Alloys and the Inversion of Order in IronChromium Alloys. Phys. Rev. Lett. 95(7), 075702 (2005)

34. Stukowski A, Sadigh B, Erhart P, et al. Efficient implementation of the concentration-dependent embedded atom method for molecular-dynamics and Monte-Carlo simulations.J Information Organizational Sci. 17(7):075005 (2009).

35. Zhou X, Johnson R, Wadley H. Misfit-energy-increasing dislocations in vapor-deposited $\mathrm{CoFe} / \mathrm{NiFe}$ multilayers.Phys Rev B. 69(14):144113 (2004).

36. M.I. Baskes, Modified embedded-atom potentials for cubic materials and impurities. Phys. Rev. B 46, 2727-2742 (1992)

37. Gullett P M, Wagner G, Slepoy A. Numerical tools for atomistic simulations. Sandia Report. (2004).

38. Byeong-Joo Lee, M. I. Baskes. Second nearest-neighbor modified embedded-atom-method potential. Phys Rev B. 62, 8564-8567 (2000).

39. Byeong Joo Lee, M.I. Baskes, Hanchul Kim, et al. Second nearest-neighbor modified embedded atom method potentials for bcc transition metals. Phys Rev. 64, 184102 (2001).
40. S.M. Valone, M.I. Baskes, R.L. Martin, Atomistic model of helium bubbles in gallium-stabilized plutonium alloys. Phys. Rev. B 73, 214209 (2006)

41. Guofeng Wang, M. A. Van Hove, P. N. Ross, et al. Monte Carlo simulations of segregation in Pt-Re catalyst nanoparticles. J Chem Phys. 121:5410 (2004).

42. James F. Ziegler, Jochen P. Biersack. The Stopping and Range of Ions in Matter. In: Bromley D.A. (eds) Treatise on Heavy-Ion Science. Springer, Boston, MA (1985).

43. Hossein Talebi, Mohammad Silani, Stéphane P. A. Bordas, Pierre Kerfriden, Timon Rabczuk. A computational library for multiscale modeling of material failure. Computational Mechanics. 53(5):1047-1071 (2014)

44. P.R. Budarapu, R. Gracie, S.-W. Yang, X. Zhuang, T. Rabczuk, Efficient coarse graining in multiscale modeling of fracture. Theoret. Appl. Fract. Mech. 69, 126-143 (2014)

45. H.-S. Jang, D. Seol, B.-J. Lee, Modified embedded-atom method interatomic potentials for $\mathrm{Mg}-\mathrm{Al}-\mathrm{Ca}$ and $\mathrm{Mg}-\mathrm{Al}-\mathrm{Zn}$ ternary systems. J Magnesium Alloys. 9(1), 317-335 (2021)

46. Peter Mahler Larsen, Søren Schmidt, Jakob Schiøtz. Robust structural identification via polyhedral template matching. Modelling and Simulation in Materials Science and Engineering. 24:055007 (2016)

47. Thomas Bonald, Bertrand Charpentier, Alexis Galland, et al. Hierarchical Graph Clustering using Node Pair Sampling. arXiv:1806. 01664.

48. Jason F. Panzarino, Timothy J. Rupert. Tracking Microstructure of Crystalline Materials: A Post-Processing Algorithm for Atomistic Simulations. JOM. 66, 417-428 (2014).

49. Alexander Stukowski, Vasily V Bulatov, Athanasios Arsenlis. Automated identification and indexing of dislocations in crystal interfaces. Model Simulation Mater Sci Eng 20:085007 (2012).

50. J. Dana. Honeycutt, Hans C. Andersen. Molecular dynamics study of melting and freezing of small Lennard-Jones clusters. J Phys Chem A. 91(19), 4950-4963 (1987).

51. D. Faken, H. Jónsson, Systematic analysis of local atomic structure combined with 3D computer graphics. Comput. Mater. Sci. 2(2), 279-286 (1994)

52. H. Zhang, Ou. Xiaoqin, B. Wei, S. Ni, M. Song, Strain direction dependency of deformation mechanisms in an HCP-Ti crystalline by molecular dynamics simulations. Comput. Mater. Sci. 172, $109328(2020)$

Publisher's Note Springer Nature remains neutral with regard to jurisdictional claims in published maps and institutional affiliations. 